\title{
Rat Forebrain Neurogenesis and Striatal Neuron Replacement after Focal Stroke
}

\author{
Jack M. Parent, MD, ${ }^{1}$ Zinaida S. Vexler, PhD, ${ }^{2,3}$ Chao Gong, MD, PhD,${ }^{1}$ Nikita Derugin, MA,${ }^{4}$ \\ and Donna M. Ferriero, MD ${ }^{2,3}$
}

\begin{abstract}
The persistence of neurogenesis in the forebrain subventricular zone (SVZ) of adult mammals suggests that the mature brain maintains the potential for neuronal replacement after injury. We examined whether focal ischemic injury in adult rat would increase SVZ neurogenesis and direct migration and neuronal differentiation of endogenous precursors in damaged regions. Focal stroke was induced in adult rats by 90 -minute right middle cerebral artery occlusion (tMCAO). Cell proliferation and neurogenesis were assessed with bromodeoxyuridine (BrdU) labeling and immunostaining for cell type-specific markers. Brains examined 10-21 days after stroke showed markedly increased SVZ neurogenesis and chains of neuroblasts extending from the SVZ to the peri-infarct striatum. Many BrdU-labeled cells persisted in the striatum and cortex adjacent to infarcts, but at 35 days after tMCAO only BrdU-labeled cells in the neostriatum expressed neuronal markers. Newly generated cells in the injured neostriatum expressed markers of medium spiny neurons, which characterize most neostriatal neurons lost after tMCAO. These findings indicate that focal ischemic injury increases SVZ neurogenesis and directs neuroblast migration to sites of damage. Moreover, neuroblasts in the injured neostriatum appear to differentiate into a region-appropriate phenotype, which suggests that the mature brain is capable of replacing some neurons lost after ischemic injury.
\end{abstract}

Ann Neurol 2002;52:802-813

The forebrain subventricular zone (SVZ) of adult rodents is a persistent germinative zone that generates olfactory bulb neurons throughout life. ${ }^{1-4}$ The neuronal precursors in the SVZ undergo a long-distance tangential migration to the olfactory bulb in a restricted pathway known as the rostral migratory stream (RMS). ${ }^{3}$ SVZ neuroblasts migrate along one another in chains within the RMS, and the chains are surrounded by astrocytic, tube-like structures. $^{3-5}$ The immature neurons also express characteristic markers, such as the polysialylated form of neural cell adhesion molecule (PSA-NCAM) $)^{4,6,7}$ and doublecortin $(\mathrm{DCx}),{ }^{8,9}$ as they proliferate and migrate. Similar neurogenic pathways also may persist in adult primates. ${ }^{10-12}$ The presence of neurogenesis in the adult mammalian forebrain raises the possibility that endogenous neural precursor cells could contribute to neuronal replacement after injury. ${ }^{13,14}$

Recent studies indicate that acute brain injury influences neuronal precursor behavior in the adult rodent forebrain SVZ. Various types of injury, such as aspiration or transection lesions of cortex, inflammatory or chemical demyelination, or percussion trauma, increase $S V Z$ precursor proliferation in the adult rodent forebrain. ${ }^{15-20}$ The fate of the SVZ neural precursors has been characterized after chemical demyelination and percussion trauma, and in these settings the newly generated cells differentiate into glia. ${ }^{18,20}$ In contrast, work by Fallon and colleagues showed that precursors arising from the SVZ generate neurons in the adult rat striatum after a combination of 6-hydroxydopamine lesions of substantia nigra and striatal transforming growth factor- $\alpha$ infusions. ${ }^{21}$ We recently found that forebrain SVZ neurogenesis also increases after chemoconvulsantinduced status epilepticus, and that a portion of the neuroblasts destined for the olfactory bulb exit the RMS prematurely and migrate into the injured forebrain. ${ }^{22}$

2To better understand the influence of brain injury on adult forebrain SVZ neuroblasts and the potential for neuronal replacement after stroke, we examined the effects of focal ischemic injury on SVZ neurogenesis. Focal or global cerebral ischemia has been shown to increase neurogenesis in the hippocampal dentate gyrus, ${ }^{23-25}$ another region of persistent neurogenesis ${ }^{26}$ in the adult mammalian brain. Zhang and colleagues recently found increased numbers of newly generated cells in the SVZ and olfactory bulb after injury in an adult rat model of embolic middle cerebral artery (MCA) occlusion. We induced focal infarcts in adult rats using the
From the ${ }^{1}$ Department of Neurology, University of Michigan Medical Center, Ann Arbor, MI; and Departments of ${ }^{2}$ Neurology, ${ }^{3} \mathrm{Pe}-$ diatrics and ${ }^{4}$ Neurosurgery, University of California, San Francisco, San Francisco, CA.

Received Apr 16, 2002, and in revised form Aug 16. Accepted for publication Aug 23, 2002.
Address correspondence to Dr Parent, Department of Neurology, University of Michigan Medical Center, 4412 Kresge III, 200 Zina Pitcher Place, Ann Arbor, MI 48109-0585.

E-mail: parent@umich.edu 
transient MCA suture occlusion (tMCAO) stroke model, and then examined SVZ cell proliferation and the phenotypes of newly generated cells in periinfarct regions by $5^{\prime}$-bromodeoxyuridine ( $\left.\mathrm{BrdU}\right)$ labeling and immunohistochemistry for cell type-specific markers. Our findings indicate that focal ischemia in the adult rat increases forebrain SVZ neurogenesis and leads to the generation of new neurons with appropriate regional specificity in the periinfarct neostriatum.

\section{Materials and Methods}

\section{Transient Middle Cerebral Artery Suture Occlusion Procedure}

Adult (190-260gm) male Sprague-Dawley rats (Harlan, Indianapolis, IN) were used for all experiments. The protocols were approved by the committees on animal research of the University of California, San Francisco and the University of Michigan. Rats were anesthetized initially in a chamber with $4 \%$ isoflurane in a $70 \%$ to $30 \%$ mixture of $\mathrm{N}_{2} \mathrm{O}$ to $\mathrm{O}_{2}$, and the isoflurane was decreased to 1.5 to $1.75 \%$ after induction. $\mathrm{tMCAO}$ was produced for 90 minutes as described previously. ${ }^{27}$ No blood loss occurred during occlusion or reperfusion. Sham-operated controls were anesthetized in an identical manner and underwent midline cervical incision without perturbation of the carotid arteries.

A total of 33 animals underwent $\mathrm{tMCAO}$ and 3 had sham surgery. The 3 controls and 18 rats in the tMCAO group were anesthetized with isoflurane as described above; 15 animals (including 4 used for quantification studies, see below) were anesthetized with ketamine $(80 \mathrm{mg} / \mathrm{kg})$ and xylazine $(12 \mathrm{mg} / \mathrm{kg})$. Survival and range of infarct sizes were similar with both anesthetics. Infarcts involved both striatum and cortex in 19 of 33 rats, and only these samples were used for quantitative analyses (see below). The remainder of the animals had no lesion or a small, focal lesion restricted to the striatum. None had only cortical lesions.

\section{5'-Bromodeoxyuridine Labeling and Tissue Processing}

The thymidine analog BrdU (Boehringer Mannheim, Indianapolis, IN) was used to label S-phase cells. ${ }^{28}$ Rats received two BrdU injections $(50 \mathrm{mg} / \mathrm{kg}$ IP in phosphate-buffered saline), 6 hours apart, on days 7 to 9 after tMCAO for a total of six injections. This time point was chosen based on prior studies showing maximal increases in SVZ cell proliferation at 7 days after prolonged seizures or focal ischemia, ${ }^{22,26}$ and also to limit the labeling of proliferating glial cells in the early period after brain injury. Animals used for immunofluorescence and quantitative analyses were killed $10(\mathrm{n}=5), 14(\mathrm{n}=4), 21$ $(\mathrm{n}=3)$, or $35(\mathrm{n}=4)$ days after tMCAO (days $1,5,12$, or 26 after the last BrdU injection). Additional animals were killed at 21- and 35-day time points and used for immunofluorescence double labeling. Sham-operated controls received identical $\mathrm{BrdU}$ injections and were killed 14 days after tMCAO ( $\mathrm{n}=3$ ). Rats were killed by an overdose of pentobarbital sodium (Abbott Laboratories, North Chicago, IL) and were transcardially perfused with phosphate-buffered saline followed by $4 \%$ paraformaldehyde in phosphate-buffered saline $(360 \mathrm{ml} ; \mathrm{pH} 7.4)$. Brains were postfixed overnight, cryoprotected, frozen, and microtome-sectioned coronally through the striatal SVZ, RMS, and olfactory bulb at $40 \mu \mathrm{m}$. Every sixth section was processed for Nissl stain and immunohistochemistry as described below.

\section{Nissl Stain and Peroxidase Immunohistochemistry}

Sections were Nissl stained according to standard methods. ${ }^{22}$ Diaminobenzidine peroxidase immunohistochemistry was performed on free-floating sections as described previously. ${ }^{29,30}$ To label proliferating cells, we used primary antibodies that recognize BrdU (mouse monoclonal; 1:1,000; Boehringer Mannheim) and cdc2 (mouse monoclonal; 1:1,000; Santa Cruz Biotechnology, Santa Cruz, CA). Cdc2 (p34 $\left.{ }^{\mathrm{cdc} 2}\right)$ is a cell cycle-related kinase expressed by proliferating cells in the adult rodent SVZ. ${ }^{31}$ Migrating neuroblasts were identified using an antibody to DCx (rabbit polyclonal; 1:2,000; a gift from C. Walsh, Harvard University), a microtubule-associated protein expressed by SVZ neuroblasts. ${ }^{8}$ For some experiments, we used a DCx antibody that we generated using the same protocol (Covance, Richmond, CA). ${ }^{8}$ Both DCx antibodies showed identical patterns of immunolabeling using both peroxidase and fluorescence immunohistochemistry. For BrdU immunostaining, DNA was first denatured by incubating tissue sections in $2 \mathrm{~N} \mathrm{HCl}$ for 30 minutes at $37^{\circ} \mathrm{C}$ followed by a 10 -minute wash in $0.1 \mathrm{M}$ borate solution, $\mathrm{pH} 8.5$.

\section{Immunofluorescence Histochemistry and Confocal Microscopy}

Single and double-label immunofluorescence histochemistry was performed on free-floating sections according to previously described methods. ${ }^{29,30}$ Primary antibody dilutions used were 1:200 for BrdU (rat monoclonal; Accurate Chemical, Westbury, NY); 1:1,000 for PSA-NCAM (mouse IgM 5A5 clone; Developmental Hybridoma Bank, University of Iowa); 1:1,000 for DCx; 1:200 for glial fibrillary acidic protein (GFAP; rat monoclonal; a gift from V. Lee, University of Pennsylvania), or 1:500 for GFAP (rabbit polyclonal; Sigma, St. Louis, MO); 1:200 for vimentin (mouse monoclonal; DAKO, Carpinteria, CA); 1:1,000 for myelin oligodendrocyte glycoprotein (MOG; mouse monoclonal, a gift from C. Bernard, Melbourne, Australia); 1:200 for ED1 (mouse monoclonal, Serotec, Oxford, UK); 1:500 for glucose transporter-1 (Glut-1; rabbit polyclonal; Chemicon, Temecula, CA); 1:1,000 for NeuN (mouse monoclonal, Chemicon); 1:8,000 for DARPP-32 (a gift from P. Geengard, Rockefeller Institute, New York); 1:500 for calbindin D28k (mouse monoclonal; Sigma); and 1:400 for class III $\beta$-tubulin (TuJ1 clone, mouse monoclonal; Covance). Secondary antibodies used for immunofluorescence were goat anti-rat IgG conjugated to fluorescein isothiocyanate (FITC) or Alexa 594 (1:800; Molecular Probes, Eugene, OR), goat anti-mouse IgG conjugated to FITC or Texas Red, goat anti-rabbit IgG conjugated to FITC or Alexa 594, and goat anti-mouse IgM conjugated to FITC or Texas Red (except as noted, all secondary antibodies were used at 1:400 dilutions, and were species cross-adsorbed from Jackson Immunoresearch Laboratories, West Grove, PA).

For double-label immunofluorescence using rat anti-BrdU and a second primary antibody, free-floating sections were first incubated in the second primary antibody for 24 hours at $4^{\circ} \mathrm{C}$, washed for 45 minutes, postfixed in $4 \%$ paraformal- 
dehyde for 20 minutes, and denatured as described above. After incubation in blocking solution, sections were incubated in rat anti-BrdU and the second primary antibody for another 24 hours at $4^{\circ} \mathrm{C}$. Images were obtained using a BioRad MRC 1024 (Hercules, CA) or Zeiss LSM 510 (Oberkochen, Germany) confocal laser microscope as single optical images or $z$-series stacks of 0.8 to $1 \mu \mathrm{m}$ slice thickness. Images were transferred to Adobe Photoshop (Adobe Systems, Mountain View, CA) for color merging.

\section{Data Quantification and Analysis}

For quantification of Nissl staining and cdc2, DCx, or BrdU immunostaining, images were digitally captured using a Nikon Microphot-SA (Melville, NY) or Leica DMIRB (Heidelberg, Germany) microscope under a $\times 10$ objective and attached to a JVC (Yokohama, Japan) or SPOT-RT (SPOT Diagnostic Instruments, Sterling Heights, MI) digital camera. Images were imported into NIH image 1.61 for blinded densitometric analysis. Regions of interest were drawn manually over the SVZ or adjacent neostriatum (see Fig 1A). Measurements in the regions of interest were made from three to five sections per animal in NIH image using a threshold cutoff of four standard deviations above background grayscale levels. Statistical comparisons between ipsilateral and contralateral hemispheres were made using paired $t$ tests, and between tMCAO and sham-surgery groups using two-tailed Student's $t$ tests, both with StatView software (Abacus Concepts, Berkeley, CA). Counts of BrdU- and double-labeled cells in the infarcted neostriatum were made using confocal $z$-series stacks obtained from three to five randomly chosen microscopic fields under a $\times 63$ water objective. The stacks were carefully analyzed to ensure colocalization of signal within individual cells and the exclusion of "satellite" cells. ${ }^{32}$

\section{Results}

Increased Cell Proliferation and Expansion of the Subventricular Zone after Occlusion of the Right Middle Cerebral Artery

As described previously, ${ }^{27}$ 90-minute tMCAO in adult rats typically resulted in large infarcts involving the ipsilateral striatum and neocortex at the level of the striatal SVZ (see Fig 1B). We first sought to determine whether focal ischemic injury increases SVZ cell proliferation. Brain sections were immunostained for the cell cycle antigen $\mathrm{cdc} 2\left(\mathrm{p} 34^{\mathrm{cdc} 2}\right)$, which is expressed by proliferating SVZ cells, ${ }^{31}$ between 10 and 35 days after tMCAO. Compared with the contralateral hemisphere, cdc2 immunoreactivity increased significantly ipsilateral to the stroke at 14 days after tMCAO (Figs 2A, B and 3$)$, and trended toward increases at 10 and 21 days $(p=0.1$ for each, paired $t$ test). The increase in cdc2 immunoreactivity ipsilateral to the stroke predominated in the dorsolateral SVZ in which the highest numbers of proliferating cells are normally found. ${ }^{22,31}$ By 35 days after tMCAO, ipsilateral SVZ structure was distorted (see Fig 2C and J), making quantitative comparison with the contralateral side difficult. We also determined interhemispheric differences in SVZ cell

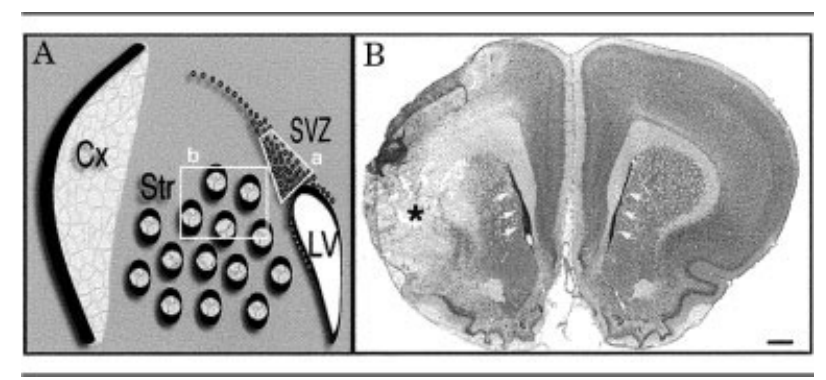

Fig 1. Schematic diagram of the striatal subventricular zone (SVZ) with areas used for quantification, and an example of a stroke in an adult rat 2 weeks after 90-minute occlusion of the right middle cerebral artery (tMCAO) (A) Diagram of a coronal view at the level of the striatal SVZ. Boxed areas show the regions of interest for measurements in the dorsolateral SVZ (a) and neostriatum (b). $L V=$ lateral ventricle; Str = striatum; $C x=$ cortex. (B) Nissl-stained, coronal section through the rostral SVZ of an adult rat 2 weeks after $T M C A O$. Note the large infarct (asterisk) and the larger size of the dorolateral SVZ ipsilateral to the stroke (arrows). Scale bar $=300 \mu \mathrm{m}$.

proliferation of stroke animals versus sham-operated controls killed at the 14 day time point. The ratio of ipsilateral to contralateral SVZ cdc2 immunostaining was significantly greater after ischemia (tMCAO group ratio 2.06 vs control ratio $1.48 ; p<0.05$, two-tailed $t$ test). To confirm the stroke-induced increase in SVZ cell proliferation, we administered $\mathrm{BrdU}$ on days 7 to 9 after tMCAO, killed animals 24 hours after the last injection and examined BrdU labeling by immunohistochemistry. In the contralateral hemisphere, BrdU labeling was largely confined to the SVZ (see Fig 2D). On the side of the stroke, however, BrdU labeling was increased in the SVZ, and labeled cells also were found in the adjacent neostriatum (see Fig 2E). Quantification of BrdU labeling at 10 days after tMCAO showed significant increases of 18 and $460 \%$ in the ipsilateral SVZ and striatum, respectively, compared with the contralateral hemisphere (see Fig 3).

We next used Nissl staining to determine whether the increase in cell proliferation ipsilateral to the infarct led to an expansion of the SVZ. As expected, the dorsolateral SVZ of the infarcted hemisphere enlarged within 2 weeks after tMCAO compared with the contralateral side and to sham-operated controls at both rostral (see Fig 1B) and more caudal (see Fig 2F-J) SVZ levels. SVZ Nissl staining was increased significantly at 14 and 21 days after tMCAO (see Fig 3). No qualitative change was seen in the cell density of the SVZ of either hemisphere after focal ischemia at these time points. As was the case for cdc2, the interhemispheric ratio of SVZ cresyl violet-stained area was greater after $\mathrm{tMCAO}$ compared with sham-operated controls (tMCAO group ratio 1.46 vs control ratio 1.09, $p<0.05$, two-tailed $t$ test). The increase in mitotic activity identified by $\mathrm{BrdU}$ and cdc2 immuno- 

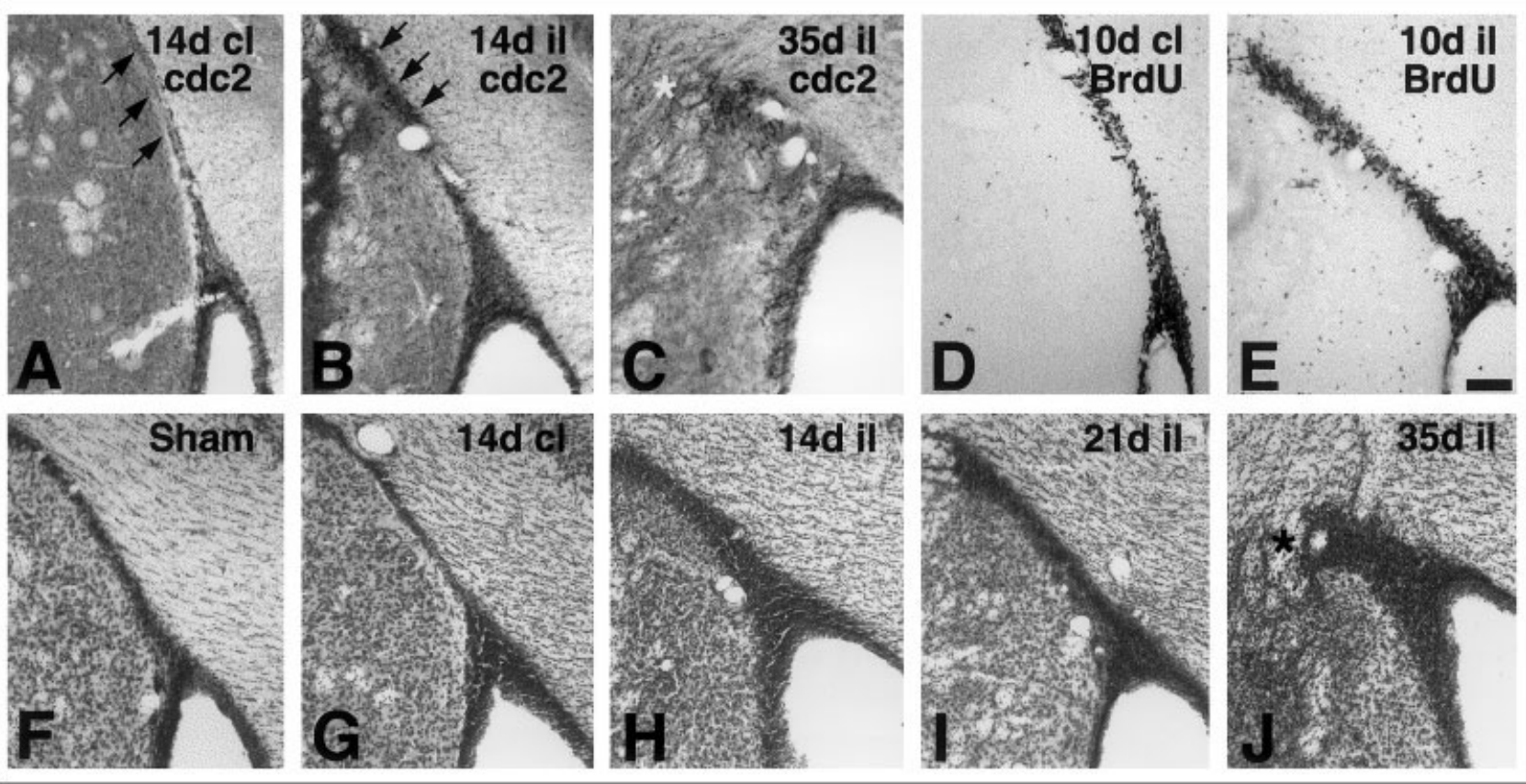

Fig 2. Coronal sections of the dorsal half of the striatal subventricular zone (SVZ) show increased cell proliferation and an enlarged $S V Z$ after focal ischemia. (A, B) Immunoreactivity for the endogenous cell proliferation marker cdc2 is increased in the dorsolateral $S V Z$ of the infarcted hemisphere (B) 2 weeks after occlusion of the right middle cerebral artery (tMCAO) compared with the contralateral (cl) hemisphere (A), especially in the infracallosal tail of the SVZ (arrows in $A, B$ ). (C) cdc2 immunostaining 35 days after $t M C A O$ shows loss of the infracallosal tail (asterisk) and some labeling in the adjacent neostriatum. (D, E) Increased 5 'bromodeoxyuridine immunoreactivity 10 days after $t M C A O$ in the SVZ, striatum, and corpus callosum ipsilateral to the stroke (E) compared with contralateral $(D)$. ( $F, G)$ Cresyl violet staining shows an expanded dorsolateral SVZ in the injured hemisphere 14 to 35 days after tMCAO (H-J) compared with contralateral $(G)$ or a sham-operated control $(F)$. At 35 days after ischemia $(J)$, the infracallosal tail is lost and the dorsolateral SVZ extends into the neostriatum (asterisk in J). Scale bar (in E) $=100 \mu m$. $c l=$ contralateral; il = ipsilateral.

staining (within 10-14 days) preceded the maximal expansion of the SVZ (within 14-21 days after tMCAO by Nissl stain), suggesting that the SVZ cells accumu- lated because of either accelerated proliferation or failure of migration out of the SVZ. However, BrdUlabeled cells were found in the olfactory bulbs of both

Fig 3. Quantification of cell proliferation and subventricular zone (SVZ) size at different time points after occlusion of the right middle cerebral artery (tMCAO). The mean area of immunoreactivity (for 5'-bromodeoxyuridine [BrdU], cdc2, or DCX) or Nissl staining (CV) in the ipsilateral (i) SVZ or neostriatum (Stm) relative to the contralateral (c) hemisphere is shown. All measurements are of the SVZ except for the second pair $(10 \mathrm{~d}$ Stm BrdU). Single asterisk, $\mathrm{p}<0.05$, double asterisk, $\mathrm{p}<0.01$ (paired $\mathrm{t}$ test). $N=3$ to 5 per group. Error bars are SEM.

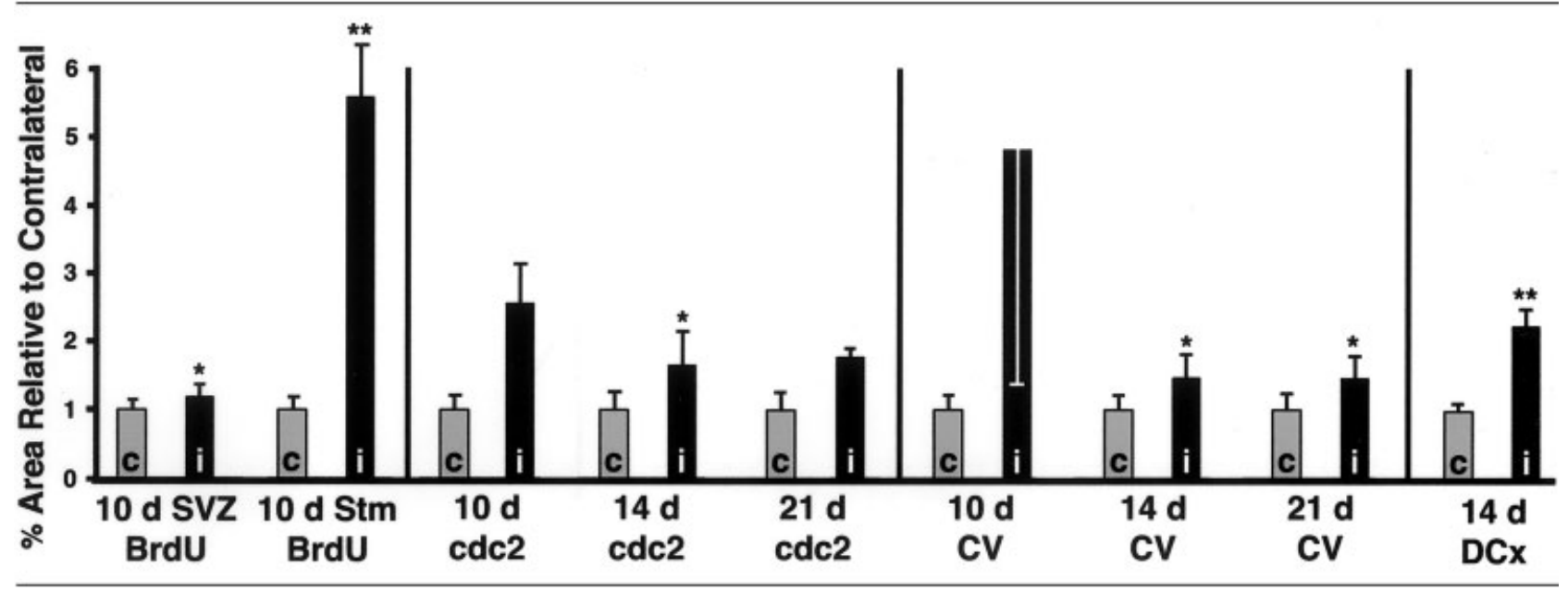




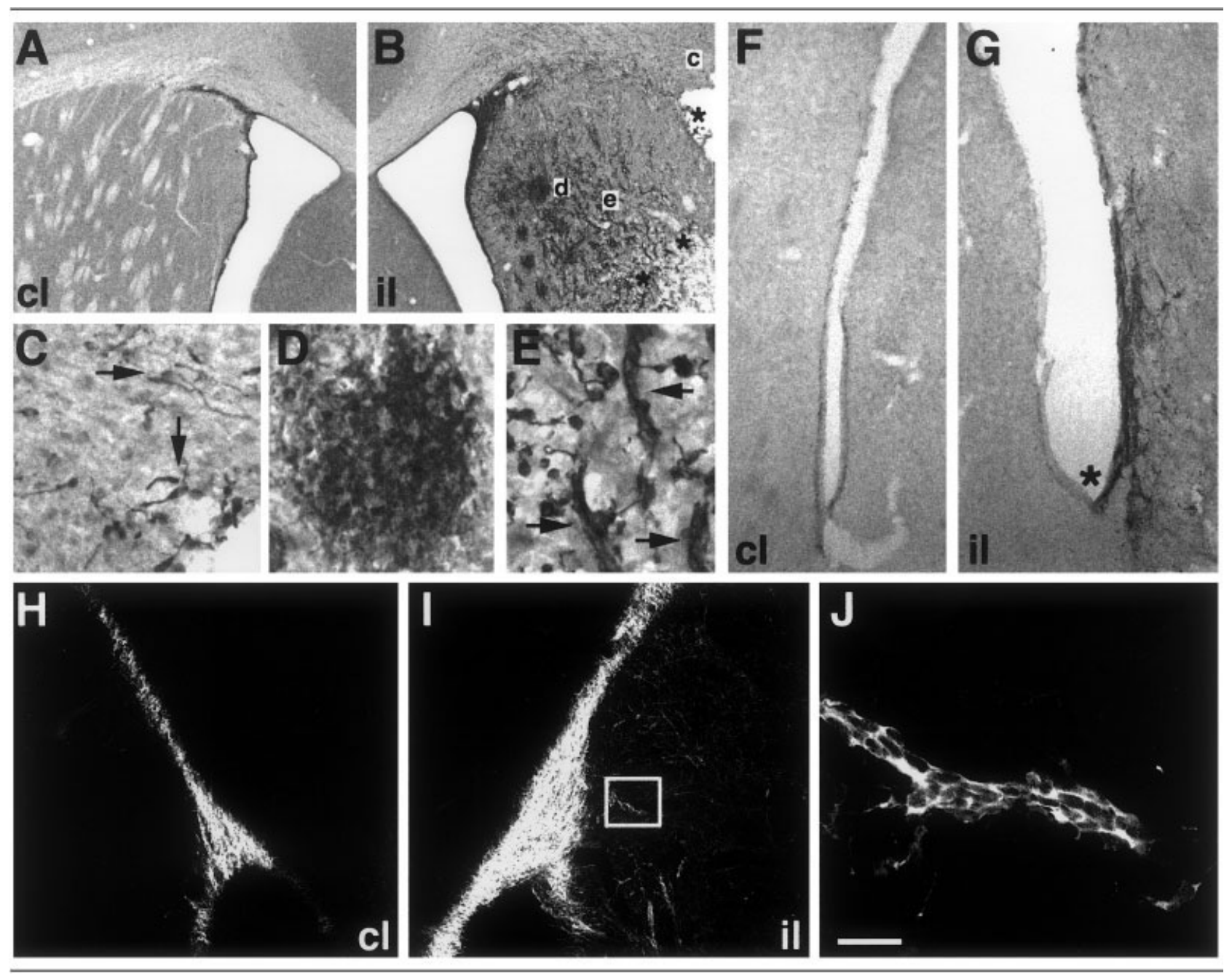

Fig 4. Subventricular zone (SVZ) doublecortin immunoreactivity after focal ischemia. $(A, B) D C x$ immunoreactivity in the ipsilateral SVZ and striatum (B) is markedly increased compared with contralateral $(A)$ in an adult rat 2 weeks after occlusion of the right middle cerebral artery (tMCAO). Asterisks in $B$ denote the border of the infarct. $(C-E)$ Higher magnification images of the boxed areas in $B$ show labeled cells with migratory morphology in periinfarct cortex ( $C$, arrows), and in the neostriatum in clusters (D) or chains ( $E$, arrows). ( $F, G) D C x$ immunoreactivity restricted to the inferior aspect of the ipsilateral SVZ of a different animal 2 weeks after focal ischemia. The asterisk in $G$ marks the inferior tip of the lateral ventricle. (H-J) Confocal images of a $D C x$-immunostained coronal section through the striatal SVZ from an adult rat 3 weeks after tMCAO. (J) Higher magnification of the boxed area in I shows DCx-positive neuroblasts extending in chains from the SVZ to the injured neostriatum. Scale bar in $J=250 \mu \mathrm{m}$ for $A, B ; 30 \mu \mathrm{m}$ for $C$ to $E ; 100 \mu \mathrm{m}$ for $F$ to I; and $20 \mu \mathrm{m}$ for J. il = ipsilateral; cl = contralateral.

Fig 5. Confocal immunofluorescence double labeling shows increased neuroblast numbers in the subventricular zone (SVZ) and neostriatum after focal stroke. (A, B) DCX-labeled cells in the SVZ 14 days after occlusion of the right middle cerebral artery (tMCAO) coexpress neuron-specific $\beta$-tubulin but not glial fibrillary acidic protein. (C) The neuroblasts also express PSA-NCAM (green) and many incorporated 5'-bromodeoxyuridine (BrdU; red) given 5 to 7 days earlier. (D-E) Double labeling for PSANCAM (red) and vimentin (green) 21 days after tMCAO. The inset in $E$ is a higher magnification view of the boxed area. PSANCAM immunoreactivity extends from the dorsolateral SVZ into the injured neostriatum (asterisk in E). (F) A higher magnification image from this region. Chains of PSA-NCAM-labeled cells (arrowheads; red) are adjacent to vimentin-immunoreactive astrocytes (green). Similar chains are seen with DCx immunostaining (arrowheads in K). (G-J) Double labeling for BrdU (red) and DCX (green in H), PSA-NCAM (green in I), or MAP2 (green in G, J) in the ipsilateral neostriatum 14 days after tMCAO. The cells in $H$ to $J$ are near the infarct boundary at approximately the boxed area in G. Arrows point to double-labeled cells. $(K-M) D C x$-expressing cells (green) in the ipsilateral neostriatum 14 days after focal ischemia coexpress MAP2 (red in M) but not vimentin (red in $K$ ) or the oligodendrocyte protein $M O G$ (red in $H$ ). Scale bar in $M=75 \mu m$ for $A$ to $C ; 100 \mu m$ for $D$, E; $125 \mu \mathrm{m}$ for $G$; and $25 \mu \mathrm{m}$ for inset in $E$ and $F$ and $H$ to $L$. 

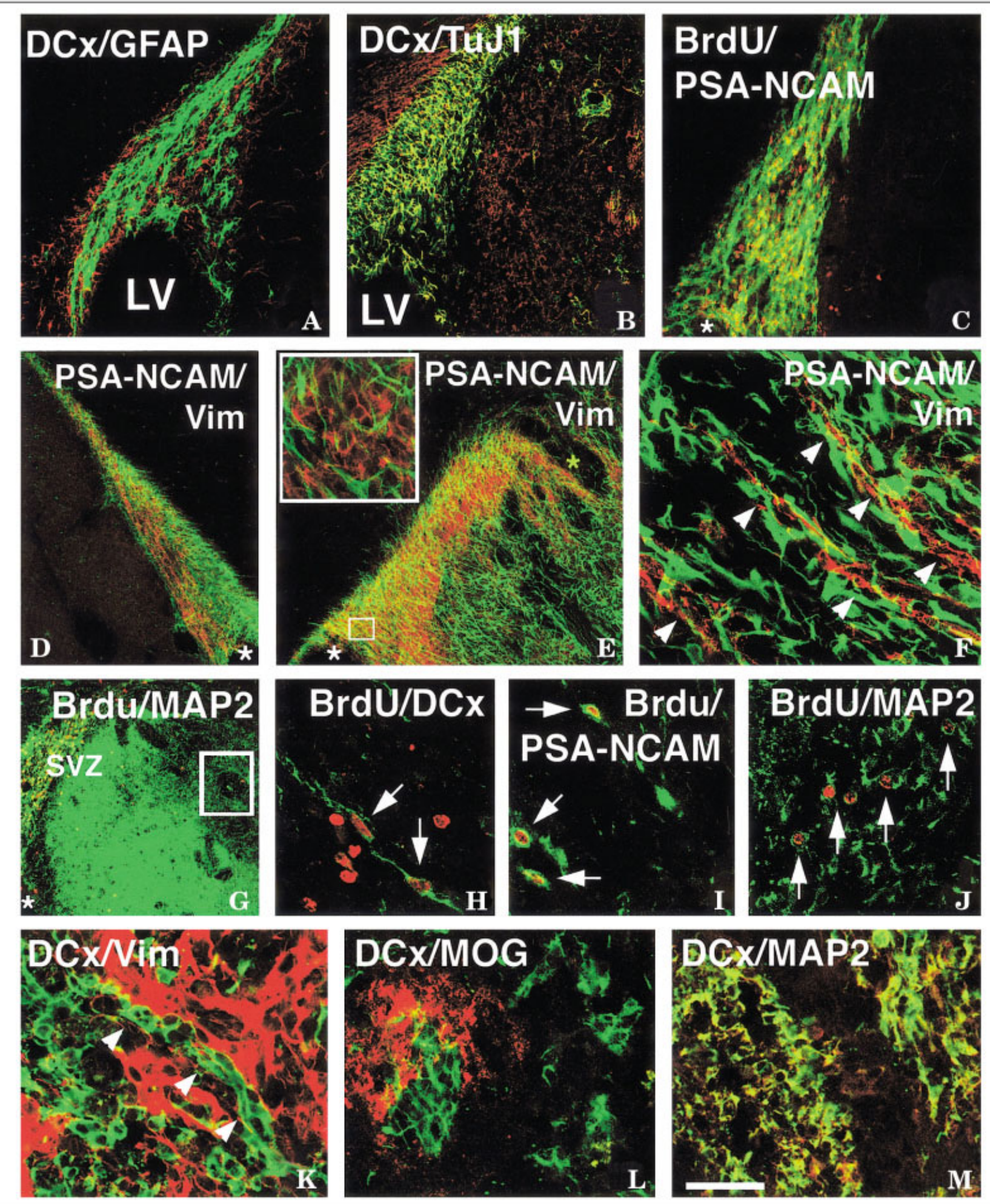

Figure 5

hemispheres at 2 to 3 weeks after tMCAO (data not shown), indicating that migration in the SVZ-olfactory bulb pathway was not impeded by the stroke.

Nissl staining 5 weeks after tMCAO showed a distortion of the dorsolateral SVZ structure in the infarcted hemisphere. The dorsolateral, infracallosal tail was lost and extended inferolaterally toward the injured neostriatum (see Fig 2J). Although cell proliferation and Nissl staining in the ipsilateral SVZ consistently increased after large infarcts involving both striatum and cortex, smaller 
ischemic injury limited to the striatum produced a smaller and more variable effect. Examination of cdc2 expression, BrdU labeling and Nissl staining in the proximal RMS showed a similar expansion ipsilateral to the infarct (data not shown), although this change was seen in only a few animals in which the infarct extended rostrally to RMS levels. Taken together, these data indicate that focal ischemic injury increases SVZ precursor proliferation and expands the forebrain SVZ.

\section{Occlusion of the Right Middle Cerebral Artery Increases Neurogenesis in the Forebrain Subventricular Zone}

To determine whether the stroke-induced SVZ expansion resulted from increased numbers of immature neurons, we examined the patterns of immunostaining for markers selectively expressed by SVZ neuroblasts. First, the expression of $\mathrm{DCx}$, a microtubule-associated protein present in migrating neuronal precursors, ${ }^{8,22}$ was examined after tMCAO. SVZ DCx immunoreactivity appeared greater in the SVZ ipsilateral to the stroke at all time points examined (Fig 4A, B, H, I). Quantification of DCx immunostaining in the SVZ at 14 days after tMCAO showed a significant increase ipsilateral to the stroke (see Fig 3). As described for cdc2 and Nissl staining, the interhemispheric ratio of DCx expression was greater 14 days after tMCAO compared with shamoperated controls (tMCAO group ratio 2.25 vs control ratio $1.12, p<0.01$, two-tailed $t$ test). In addition to ischemia-induced increases in DCx expression, we found similar increases in immunoreactivity for other markers of SVZ neuroblasts, including PSA-NCAM and neuronspecific $\beta$-tubulin (Fig 5D, E and data not shown). Comparable to the pattern of increased cell proliferation and enlargement of the SVZ, the expansion of SVZ neuroblasts after tMCAO occurred in the lateral aspect of the SVZ but was maximal in the dorsolateral region (see Fig 4). In some instances when smaller, more localized infarcts (still involving striatum and cortex) occurred, however, an increase in SVZ neuroblasts appeared focally in the lateral or inferior aspects of the SVZ adjacent to the site of injury (see Fig 4F and G). This finding suggested that lesser degrees of ischemic injury exert a more discrete effect on SVZ neuronal precursors.

To confirm that the SVZ neuroblasts were newly generated after tMCAO, we performed immunofluorescence double labeling for BrdU and immature neuronal markers. Confocal optical images were carefully examined for colocalization of labeling within cells. As expected, cells in the forebrain SVZ that had incorporated BrdU 5 to 7 days earlier also expressed the neuroblast markers PSA-NCAM, neuron-specific $\beta$-tubulin and DCx, in both ipsilateral and contralateral hemispheres, at 2 weeks after tMCAO (see Fig 5C and data not shown). To determine whether the SVZ astrocyte population also expanded after tMCAO, we immu- nostained for vimentin and GFAP, intermediate filament proteins expressed by astrocytes in the SVZ. ${ }^{33,34}$ Compared with markers of SVZ neuroblasts, expression of these astrocytic markers was relatively unchanged in the forebrain SVZ after tMCAO despite markedly increased immunoreactivity in the adjacent striatum of the infarcted hemisphere (see Fig 5D and E). A similar finding has been reported after seizure-induced injury. ${ }^{22} \mathrm{Neu}-$ roblasts in the SVZ did not coexpress astrocytic markers in either the ipsilateral or contralateral hemispheres (see Fig 5A, D, E). These data indicate that focal infarcts induced by tMCAO relatively selectively stimulate neurogenesis in the forebrain SVZ at the time points examined.

\section{Chains of Neuroblasts Extend to Periinfarct Regions after Stroke}

In a previous study, we found that seizure-induced injury altered the migration of SVZ neuroblasts in the forebrain. ${ }^{22}$ To determine whether focal ischemic injury also influences the destinations of SVZ neuroblasts that normally migrate to the olfactory bulb, we first examined the pattern of $\mathrm{BrdU}$ labeling outside the forebrain SVZ at various times after tMCAO. At all time points examined after focal ischemia, BrdU-labeled cells were found near periinfarct regions in markedly greater numbers than in homotopic areas of the uninjured hemisphere or in controls (see Figs 2D, 2E, 3, and 6). The numbers of BrdU-immunoreactive cells near infarcted striatum or cortex peaked at 14 days after stroke (see Fig 6B-D and data not shown) and persisted for at least 5 weeks after tMCAO, the latest time point examined (see Fig $6 \mathrm{D}$ and E). We next sought to determine whether neuroblasts were also present after injury in these regions that normally do not support neurogenesis. We immunostained for DCx and PSA-NCAM and compared the expression patterns in the infarcted hemisphere with those of the contralateral side at 14 to 21 days after tMCAO. Many DCx-expressing cells with migratory profiles were present in periinfarct regions outside of the SVZ but were nearly completely absent in homologous regions of the noninfarcted hemisphere or in shamoperated controls (see Fig 4, and data not shown). These cells appeared at all time points examined after tMCAO (between 10 and 35 days) and were maximal at 14 days after the stroke. At this time point, DCx immunostaining was more than 25 -fold higher in the injured striatum compared with the contralateral hemisphere (mean percentage of striatal area immunostained \pm standard error of the mean in the ipsilateral hemisphere $0.593 \pm$ 0.149 vs $0.022 \pm 0.01$ in the contralateral side; $p=$ 0.03 , paired $t$ test). Similar differences were found in the pattern of striatal PSA-NCAM immunostaining after stroke (see Fig 5D-F).

We suspected that the cells near injured regions expressing DCX and other neuroblast markers were immature neurons. These markers, however, occasion- 
ally are expressed by mature neurons putatively involved in network reorganization. ${ }^{9}$ To confirm that these cells were indeed newly generated, we used BrdU labeling and double-label immunofluorescence for cell type-specific markers. As expected, many of the BrdU-labeled cells found outside of the SVZ in the periinfarct neostriatum 2 to 3 weeks after tM$\mathrm{CAO}$ (and 5-12 days after the last BrdU injection) coexpressed the immature neuronal markers $\mathrm{DCx}$, PSA-NCAM, and MAP2C (see Fig 5G-J). The double-labeled cells typically exhibited morphologies consistent with migrating neuroblasts, including elongated cell bodies and bipolar processes. Some BrdUimmunoreactive cells in the injured striatum also expressed the endothelial cell marker Glut-1 or, less frequently, astrocytic (GFAP) or activated microglial (ED1) markers at 14 to 21 days after tMCAO (data not shown). The DCx- or PSA-NCAM-immunostained cells in periinfarct regions were confirmed to be neuronal as they coexpressed neuron-specific $\beta$-tubulin or MAP2, but not glial or endothelial cell markers (see Fig 5B, F, K-M, and data not shown).

Fig 6. 5'-Bromodeoxyuridine (BrdU)-labeled cells in the striatum and cortex after focal ischemia. (A, B) BrdU immunostaining 2 weeks after occlusion of the right middle cerebral artery (tMCAO). (C, D) Some BrdU-labeled cells persist in the periinfarct neostriatum at $21(C)$ and 35 (D) days after focal ischemia. The dorsolateral tip of the subventricular zone (SVZ) is just outside the upper left of the panels. (E) BrdU-positive cells in the periinfarct frontal neocortex at 5 weeks after $t M$ $C A O$ (arrows point to the infarct border). Lateral is to the right in $B$ to $E$. $L V=$ lateral ventricle; il $=$ ipsilateral to stroke; $c l=$ contralateral to stroke. Scale bar in $A=200 \mu m$ for $A$ and $B$ and $100 \mu m$ for $C$ to $E$.

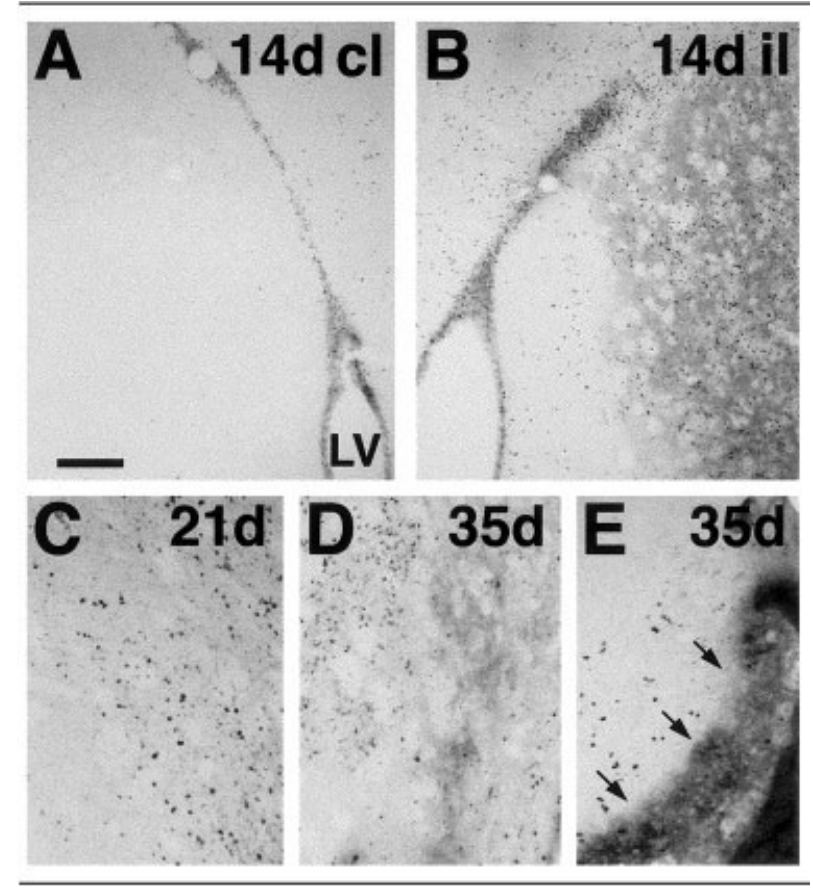

DCx-immunoreactive cells sometimes appeared as individual migratory profiles near infarcted cortex, or in peculiar cell clusters in the neostriatum in which many of the cells coexpressed neuronal, but not glial, markers (see Figs 4B-D and 5L and M). The precise nature of the cell clusters was uncertain; however, they were composed predominantly of neurons based on double labeling, and they appeared to "avoid" the neostriatal white matter tracts. Interestingly, DCx- or PSA-NCAM-immunoreactive cells frequently were observed in chains extending laterally or inferolaterally from the SVZ to the injured neostriatum (see Figs 4 and 5E, F, K). The chains appeared very similar to the chains of migrating neuroblasts seen normally in the rodent RMS. They arose from lateral or dorsolateral SVZ regions of the infarcted hemisphere and were typically in close contact with astroctyes, which were present in markedly increased numbers in the injured hemisphere (see Fig 5D-F, K). These data suggest that newly generated neuroblasts migrate from the SVZ to regions of ischemic brain injury, perhaps guided by astrocytes activated after injury.

\section{Newly Generated Neurons in the Neostriatum Differentiate into a Region-appropriate Phenotype} and Persist at Least 5 Weeks after Ischemia The finding of chains of neuroblasts extending from the forebrain SVZ to periinfarct regions suggested that cues induced by focal ischemic injury promote ectopic neurogenesis in the adult rat forebrain. However, newly generated neurons are likely to be a useful source for neuronal replacement after injury only if they are able to survive and differentiate into neuronal subtypes appropriate to the injured region. To determine whether newly generated cells persist near damaged areas, we first examined BrdU labeling in the injured hemisphere at 5 weeks after tMCAO. At the 5-week time point (26 days after the last BrdU injection), we found BrdU-immunoreactive cells in periinfarct regions of striatum and cortex (see Fig 6D and E). The number of BrdU-labeled cells at 5 weeks after stroke was visibly less than earlier time points (see Fig 6B-E), suggesting that many of the newly generated cells died over time. It is also possible that cells which previously incorporated BrdU divided a sufficient number of times that the BrdU content in their progeny was diluted to levels below immunocytochemical detection.

We next sought to determine the phenotypes of the BrdU-labeled cells that persisted in regions adjacent to ischemic injury. Immunofluorescence double labeling was performed at 5 weeks after $\mathrm{tMCAO}$, and confocal $z$-series images were collected and carefully examined for colocalization of labeling within cells. BrdU-labeled cells near infarcted cortex did not express the neuronal markers NeuN and MAP2 but occasionally expressed markers of astrocytic or endothelial lineage cells (data not 
shown). In regions adjacent to infarcted neostriatum 5 weeks after tMCAO, however, a large portion of the BrdU-labeled cells exhibited neuronal morphologies and was immunoreactive for the neuronal nuclear antigen NeuN (see Fig 7A and B). To determine whether these newly generated neurons expressed a phenotype appropriate for their location in the injured neostriatum, we double-labeled for BrdU and DARPP-32, calbindin D28k, or parvalbumin. Thirty-five days after tMCAO, many cells double-labeled for BrdU and DARPP-32 or calbindin (see Fig 7C and D), markers of neostriatal medium spiny neurons. ${ }^{35,36}$ No BrdU-immunoreactive cells expressed parvalbumin (data not shown), a marker for a subset of striatal interneurons. ${ }^{37}$ Fewer cells that had incorporated BrdU expressed GFAP or Glut-1 in the injured neostriatum (data not shown). We counted the percentages of BrdU-immunoreactive cells that coexpressed cell type-specific markers in confocal $z$-series stacks $(0.8-1.0 \mu \mathrm{m}$ optical slices) from three to five microscopic fields ( $\times 63$ objective) using two animals killed 35 days after $\mathrm{tMCAO}$. In the periinfarct striatum at 35 days after tMCAO, the percentage of BrdU-labeled cells that double-labeled for each marker was $70 \%$ for DARPP-32 (52/74 total BrdU-positive cells); 50\% for calbindin-D28k (45/91 cells); 48\% for NeuN (32/67 cells); $22 \%$ for GFAP (22/101 BrdU-labeled cells); and
$16 \%$ for Glut-1 (8/49 cells). Most of the neostriatal neurons that incorporated $\mathrm{BrdU}$ (see Fig 7) were near the boundary of the infarct.

We also found DARPP-32 expression in both ipsilateral and contralateral SVZ-olfactory bulb pathways (data not shown). This finding was unexpected given the presumed specificity of this marker for striatal projection neurons. ${ }^{35}$ At 14 days after tMCAO (5 days after the last BrdU injection), DARPP-32 expression was not present in the olfactory bulb granule cell layer in which most BrdU-labeled cells appeared, suggesting that its expression declined rapidly as the olfactory bulb neurons became postmitotic. It is possible that some of the newly generated, DARPP-32-expressing neurons in the striatum 35 days after tMCAO had not yet differentiated. This number is probably low, however, given the timing after BrdU injections and our finding that most of the cells had a differentiated neuronal morphology. Similar, albeit lower, percentages of BrdU-labeled cells in striatum also expressed the mature neuronal markers calbindin-D28k and NeuN (see Fig 7). No DARPP-32 was expressed in injured cortex after stroke (data not shown). Together, these data indicate that newly generated neurons persist in the injured neostriatum, where they appear to differentiate into a region-appropriate

Fig 7. Newly generated neurons persist in the neostriatum after occlusion of the right middle cerebral artery (tMCAO) and differentiate into a region-appropriate phenotype. (A, B) Confocal microscopic images of the periinfarct neostriatum double-labeled for 5'bromodeoxyuridine (BrdU; red) and NeuN (green). The low-magnification view (A) shows the infarct (asterisk) laterally and the lateral ventricle (LV) medially. A partial z-series from the boxed region in $A$ is shown in B. Approximately half of the BrdUlabeled cells coexpress NeuN (arrows), whereas other cells are immunoreactive only for NeuN or BrdU (arrowheads). Many BrdUlabeled cells (red) from the same periinfarct region (asterisk at the stroke border in C) also coexpress calbindin-D28K (C) or DARPP-32 (D). Arrowheads point to single-labeled cells. The spacing is $1 \mu \mathrm{m}$ between panels in $B$ and D. Scale bar in $A=$ $150 \mu \mathrm{m}$, and in $D=10 \mu \mathrm{m}$ for $B$ to $D$.
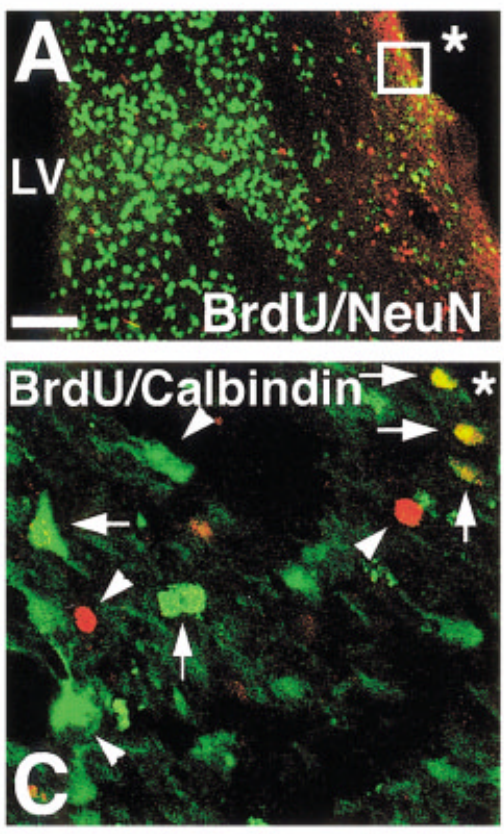
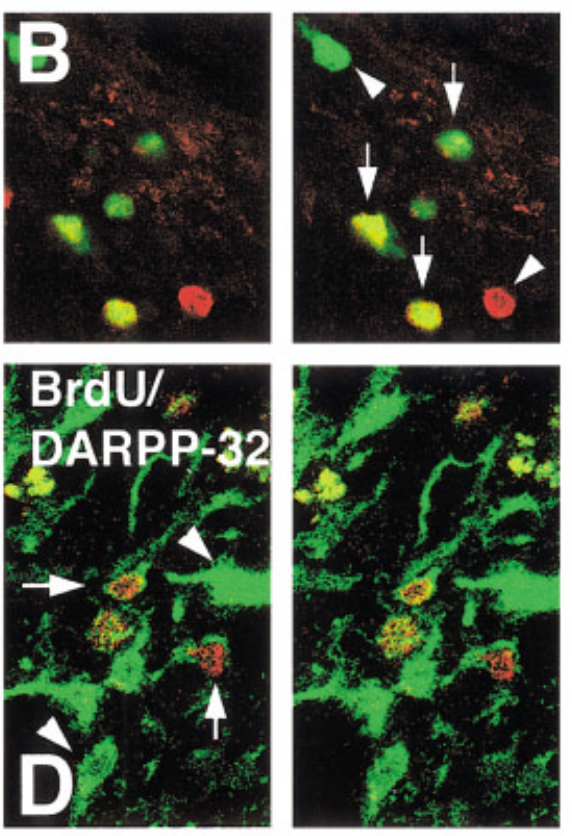
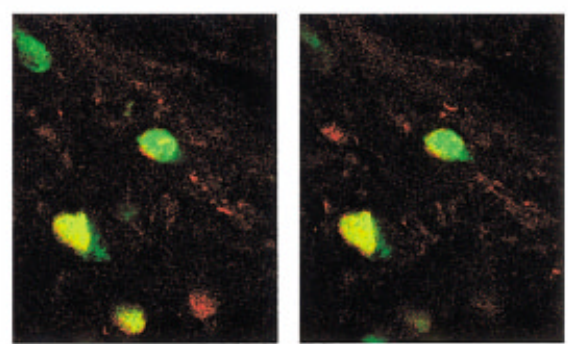

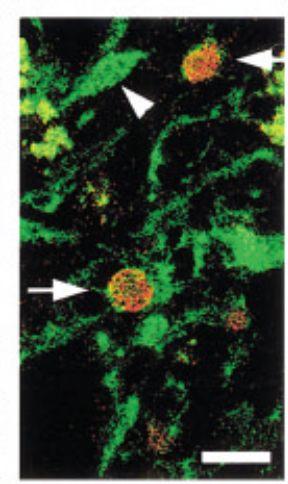


neuronal subtype corresponding to the majority of neurons lost in the striatum after tMCAO.

\section{Discussion}

This study demonstrates that adult rat forebrain neural precursors generate neurons with appropriate regional specificity after stroke. We have found that $\mathrm{MCAO}$ increases SVZ cell proliferation and neurogenesis ipsilateral to infarcted brain regions, with many SVZ neuroblasts extending ectopically to sites of injury. Importantly, in damaged neostriatum, neuroblasts differentiate into cells that have neuronal morphologies and express markers specific for medium spiny neostriatal neurons. ${ }^{35,36}$ If these new neurons are capable of long-term survival and integration, then augmentation of endogenous neurogenesis is a plausible strategy for brain regenerative therapies.

The increase in proliferating SVZ neuroblasts after focal ischemia may reflect several effects of ischemic injury on neuronal precursors. Increased neurogenesis can result from accelerated neuroblast proliferation, an enhancement of neuroblast survival, or a combination of the two. Although we cannot differentiate between these possibilities at present, our data indicate that focal ischemia induces a robust expansion of neuroblast numbers in the adult rat forebrain SVZ. Our finding of continued migration of BrdU-labeled SVZ precursors to the olfactory bulb after tMCAO excludes the possibility that an apparent increase in neurogenesis is instead caused by an accumulation of neuroblasts that fail to migrate after ischemic injury. Prior studies also have found increased cell proliferation and neurogenesis in the adult rat forebrain SVZ after focal ischemia. ${ }^{25,26}$ However, in these reports the differentiation and survival of neuroblasts outside of the SVZ-olfactory bulb pathway was not seen. The reason for the disparity from our findings may relate to differences in infarct size ${ }^{25}$ or the nature of the stroke model used. ${ }^{26}$

Neural precursors in the SVZ are a potential source of new neurons after injury. However, dormant progenitors with neurogenic potential in vitro exist throughout the adult rodent forebrain. ${ }^{38}$ The possibility exists that both sources contribute to the neostriatal neurogenesis that occurs after tMCAO. Several factors lead us to conclude that a substantial portion of the newly generated neostriatal neurons arise from the forebrain SVZ. First, the neuroblast population in the SVZ markedly expands after focal ischemia. Second, most neuroblasts are found in the striatum close to the SVZ, despite the fact that more proliferating cells in the intact adult rodent brain appear in cortex and corpus callosum than in striatum. ${ }^{38}$ Finally, we found chains of neuroblasts, in close apposition to astrocytes, extending from the SVZ to the injured striatum after focal ischemia. This structural arrangement is very similar to the chain migration of neuroblasts through astrocytic tubes characteristic of the
$\mathrm{RMS}^{3-5}$ and suggests that cues induced by ischemic injury may redirect some of the SVZ neuroblasts to migrate into periinfarct brain regions. The ectopic migration of SVZ neuroblasts into injured forebrain after prolonged seizures also has been reported recently. ${ }^{22}$ Our results do not exclude a contribution to ischemiainduced neostriatal neurogenesis from otherwise "dormant" progenitors outside the SVZ.

The molecular cues underlying ischemia-induced neurogenesis and altered neuroblast migration are unknown. Cell death may act directly to increase neurogenesis or induce the differentiation of neurons in ectopic locations. $^{39-42}$ The targeted induction of focal apoptosis in adult songbird brain increases neurogenesis, ${ }^{41}$ and in adult mouse neocortex it induces neuronal production in this otherwise dormant brain region. ${ }^{42}$ These effects may relate to trophic molecules released by neighboring cells. ${ }^{43}$ Because a portion of neuronal death after ischemia occurs via apoptotic pathways, ${ }^{44,45}$ a similar mechanism may act to stimulate neurogenesis after experimental stroke. Focal ischemia also induces a marked astrocyte proliferation (see Fig 5E), and there is evidence that astrocytes can stimulate adult rodent SVZ neural precursor proliferation in vitro. ${ }^{46}$ The activation of astrocytes after injury also may underlie the ectopic migration of SVZ neuroblasts toward damaged brain regions because a glial-derived factor can increase the migration rate of neuroblasts in explant cultures of the neonatal rat SVZ-olfactory bulb pathway. ${ }^{47}$

As expected, we found markedly increased numbers of astrocytes in brain regions near the ischemic injury. Chains of neuroblasts extending ectopically from the SVZ into the neostriatum after tMCAO were in close contact with astrocytes (see Fig 5E, F, K). This structural arrangement suggests that activated astrocytes may induce or guide the migration of SVZ neuroblasts to injury, similar to findings of astrocyte activation and altered neuronal precursor migration in the SVZ-olfactory bulb pathway after seizure-induced injury or transection lesions of the RMS. ${ }^{22,48}$ Importantly, the modulation of SVZ neuroblast proliferation or migration by glia (including astrocytes and microglia) may explain why diverse brain insults exert similar effects on SVZ neural precursors.

The injured striatum supported the neuronal differentiation of SVZ precursors after ischemic injury. The only other report of injury-induced striatal neurogenesis in adult rat, after 6-hydroxydopamine lesions of the nigrostriatal pathway, required the simultaneous administration of transforming growth factor- $\alpha{ }^{21}$ Adult striatal neurogenesis also occurred after exogenous brain-derived neurotrophic factor (BDNF) intraventricular infusion or adenoviral-mediated expression of BDNF in ependyma. ${ }^{49,50}$ However, BDNF did not induce substantial neocortical neurogenesis in these studies. Focal ischemia has been shown to increase the 
expression of BDNF and other growth or neurotrophic factors in adult rat cortex or neostriatum near the rostral SVZ or RMS. ${ }^{51-56}$ In some instances, increased expression is sustained for up to 14 days, similar to the temporal pattern of ischemia-induced neurogenesis and neuroblast migration described herein. As with increased BDNF expression, proliferating cells in injured cortex failed to differentiate into neurons after focal ischemia. Together, these findings suggest that the injured cortex is a nonpermissive environment for neuronal differentiation, either because of lack of instructive or survival cues or the presence of signals inhibitory for neurogenesis.

Stroke is a devastating neurological disorder, and those that experience cerebral infarcts show varying degrees of recovery. Existing evidence suggests that synaptic reorganization and activation of ipsilateral pathways are important for functional improvement (for reviews see Hallett ${ }^{57}$ and Rijntjes and Weiller ${ }^{58}$ ). However, our finding of ischemia-induced neuronal recruitment in the adult mammalian forebrain offers a new perspective on plasticity and the potential for neuronal replacement after brain injury. The marked extent of ectopic forebrain neurogenesis in the first few weeks after ischemic injury suggests that a large capacity for self-repair persists in adult rodent brain. Our data and the work of others ${ }^{26}$ indicate that many of these neuroblasts fail to survive after focal ischemic injury. Although augmentation of neurogenesis, including neuronal differentiation and survival, awaits a better understanding of neural stem cell biology, such manipulations eventually may lead to novel strategies to improve recovery after stroke. The functional integration of newly generated neurons in the neostriatum is a key issue that remains to be determined. Recent studies of focally induced apoptosis in adult mouse neocortex indicate that some newly generated neurons in the adult are able to survive for prolonged periods and send appropriate long-distance projections. ${ }^{42}$ Therefore, integration of new neurons into mature networks after brain injury is possible.

\section{Note Added in Proof}

Findings similar to the present study were published while this manuscript was in press. ${ }^{59}$

This study was supported by National Institutes of Health grants (NS42143, J.M.P.; NS35902, D.M.F.).

We thank Drs C. Walsh, V. Lee, P. Greengard, and C. Bernard for providing antibodies, and Dr F. Silverstein for helpful comments on the manuscript.

\section{References}

1. Altman J, Das GD. Autoradiographic and histological evidence of postnatal hippocampal neurogenesis in rats. J Comp Neurol 1965;124:319-335.
2. Lois C, Alvarez-Buylla A. Long-distance neuronal migration in the adult mammalian brain. Science 1994;264:1145-1148.

3. Lois C, García-Verdugo JM, Alvarez-Buylla A. Chain migration of neuronal precursors. Science 1996;271:978-981.

4. Thomas LB, Gates MA, Steindler DA. Young neurons from the adult subependymal zone proliferate and migrate along an astrocyte, extracellular matrix-rich pathway. Glia 1996;17: $1-14$.

5. Peretto P, Merighi A, Fasolo A, Bonfanti L. Glial tubes in the rostral migratory stream of the adult rat. Brain Res Bull 1997; 42:9-21.

6. Bonfanti L, Theodosis DT. Expression of polysialylated neural cell adhesion molecule by proliferating cells in the subependymal layer of the adult rat, in its rostral extension and in the olfactory bulb. Neuroscience 1994;62:291-305.

7. Doetsch F, Alvarez-Buylla A. Network of tangential pathways for neuronal migration in adult mammalian brain. Proc Natl Acad Sci USA 1996;93:14895-14900.

8. Gleeson JG, Lin PT, Flanagan LA, Walsh CA. Doublecortin is a microtubule-associated protein and is expressed widely by migrating neurons. Neuron 1999;23:257-271.

9. Nacher J, Crespo C, McEwen BS. Doublecortin expression in the adult rat telencephalon. Eur J Neurosci 2001;14:629_ 644.

10. Gould E, Reeves AJ, Graziano MSA, Gross CG. Neurogenesis in the neocortex of adult primates. Science 1999;286: $548-552$.

11. Pencea V, Bingaman KD, Freedman LJ, Luskin MB. Neurogenesis in the subventricular zone and rostral migratory stream of the neonatal and adult primate forebrain. Exp Neurol 2000; 172:1-16.

12. Kornack DR, Rakic P. The generation, migration, and differentiation of olfactory neurons in the adult primate brain. Proc Natl Acad Sci USA 2001;98:4752-4777.

13. O'Leary DD. Adding neurons to the adult mammalian brain. Proc Natl Acad Sci USA 1993;90:2101-2102.

14. Lowenstein DH, Parent JM. Brain, heal thyself. Science 1999; 283:1126-1127.

15. Willis P, Berry M, Riches AC. Effects of trauma on cell production in the subependymal layer of the rat neocortex. Neuropathol Appl Neurobiol 1976;2:377-388.

16. Weinstein DE, Burrola P, Kilpatrick TJ. Increased proliferation of precursor cells in the adult rat brain after targeted lesioning. Brain Res 1996;743:11-16.

17. Szele FG, Chesselet M. Cortical lesions induce an increase in cell number and PSA-NCAM expression in the subventricular zone of adult rats. J Comp Neurol 1996;368:439-454.

18. Holmin S, Almqvist P, Lendahl U, Mathiesen T. Adult nestinexpressing subependymal cells differentiate to astrocytes in response to brain injury. Eur J Neurosci 1997;9:65-75.

19. Calzà L, Giardino L, Pozza M, et al. Proliferation and phenotype regulation in the subventricular zone during experimental allergic encephalomyelitis: in vivo evidence of a role for nerve growth factor. Proc Natl Acad Sci USA 1998;95:3209-3214.

20. Nait-Oumesmar B, Decker L, Lachapelle F, et al. Progenitor cells of the adult mouse subventricular zone proliferate, migrate and differentiate into oligodendrocytes after demyelination. Eur J Neurosci 1999;11:4357-4366.

21. Fallon J, Reid S, Kinyamu R, et al. In vivo induction of massive proliferation, directed migration, and differentiation of neural cells in the adult mammalian brain. Proc Natl Acad Sci USA 2000;97:14686-14691.

22. Parent JM, Valentin VV, Lowenstein DH. Prolonged seizures increase proliferating neuroblasts in the adult rat subventricular zone-olfactory bulb pathway. J Neurosci 2002;22: $3174-3188$. 
23. Liu J, Solway K, Messing RO, Sharp FR. Increased neurogenesis in the dentate gyrus after transient global ischemia in gerbils. J Neurosci 1998;18:7768-7778.

24. Takagi Y, Nozaki K, Takahashi J, et al. Proliferation of neuronal precursor cells in the dentate gyrus is accelerated after transient forebrain ischemia in mice. Brain Res 1999;831:283-287.

25. Jin K, Minami M, Lan JQ, et al. Neurogenesis in dentate subgranular zone and rostral subventricular zone after focal cerebral ischemia in the rat. Proc Natl Acad Sci USA 2001;98: $4710-4715$

26. Zhang RL, Zhang ZG, Zhang L, Chopp M. Proliferation and differentiation of progenitor cells in the cortex and the subventricular zone in the adult rat after focal cerebral ischemia. Neuroscience 2001;105:33-41.

27. Longa EZ, Weinstein PR, Carlson S, Cummins R. Reversible middle cerebral artery occlusion without craniectomy in rats. Stroke 1989;20:294-297.

28. Miller MW, Nowakowski RS. Use of bromodeoxyuridineimmunohistochemistry to examine the proliferation, migration and time of origin of cells in the central nervous system. Brain Res 1988;457:44-52.

29. Parent JM, Yu TW, Leibowitz RT, et al. Dentate granule cell neurogenesis is increased by seizures and contributes to aberrant network reorganization in the adult rat hippocampus. J Neurosci 1997; 17:3727-3738.

30. Parent JM, Tada E, Fike JR, Lowenstein DH. Inhibition of dentate granule cell neurogenesis with brain irradiation does not prevent seizure-induced mossy fiber synaptic reorganization in the rat. J Neurosci 1999;19:4508-4519.

31. Okano HJ, Pfaff DW, Gibbs RB. RB and Cdc2 expression in brain: correlations with $3 \mathrm{H}$-thymidine incorporation and neurogenesis. J Neurosci 1993;13:2930-2938.

32. Kuhn HG, Winkler J, Kempermann G, et al. Epidermal growth factor and fibroblast growth factor- 2 have different effects on neural progenitors in the adult rat brain. J Neurosci 1997; 17:5820-5829.

33. Peretto P, Merighi A, Fasolo A, Bonfanti L. The subependymal layer in rodents: a site of structural plasticity and cell migration in the adult mammalian brain. Brain Res Bull 1999;49: 221-243.

34. Doetsch F, Caillé I, Lim DA, et al. Subventricular zone astrocytes are neural stem cells in the adult mammalian brain. Cell 1999;97:703-716.

35. Ouimet CC, Greengard P. Distribution of DARPP-32 in the basal ganglia: an electron microscopic study. J Neurocytol 1990;19:39-52.

36. Liu FC, Graybiel AM. Heterogeneous development of calbindin-D28k expression in the striatal matrix. J Comp Neurol 1992;320:304-322.

37. Kawaguchi Y, Wilson CJ, Augood SJ, Emson PC. Striatal interneurones: chemical, physiological and morphological characterization. Trends Neurosci 1995;18:527-535.

38. Palmer TD, Markakis EA, Willhoite AR, et al. Fibroblast growth factor- 2 activates a latent neurogenic program in neural stem cells from diverse regions of the adult CNS. J Neurosci 1999;19:8487-8497.

39. Gould E, McEwen BS. Neuronal birth and death. Curr Opin Neurobiol 1993;3:676-682.

40. Biebl M, Cooper CM, Winkler J, Kuhn HG. Analysis of neurogenesis and programmed cell death reveals a self-renewing capacity in the adult rat brain. Neurosci Lett 2000;291: $17-20$.

41. Scharff C, Kirn JR, Grossman M, et al. Targeted neuronal death affects neuronal replacement and vocal behavior in adult songbirds. Neuron 2000;25:481-492.
42. Magavi SS, Leavitt BR, Macklis JD. Induction of neurogenesis in the neocortex of adult mice. Nature 2000;405:951-955.

43. Wang Y, Sheen VL, Macklis JD. Cortical interneurons upregulate neurotrophins in vivo in response to targeted apoptotic degeneration of neighboring pyramidal neurons. Exp Neurol 1998;154:389-402.

44. Choi D. Ischemia-induced neuronal apoptosis. Curr Opin Neurobiol 1996;6:667-672.

45. Pettmann B, Henderson CE. Neuronal cell death. Neuron 1998;20:633-647.

46. Lim DA, Alvarez-Buylla A. Interaction between astrocytes and adult subventricular zone precursors stimulates neurogenesis. Proc Natl Acad Sci USA 1999;6:7526-7531.

47. Mason HA, Ito S, Corfas G. Extracellular signals that regulate the tangential migration of olfactory bulb neuronal precursors: inducers, inhibitors, and repellents. J Neurosci 2001;21: $7654-7663$.

48. Alonso G, Prieto M, Chauvet N. Tangential migration of young neurons arising from the subventricular zone of adult rats is impaired by surgical lesions passing through their natural migratory pathway. J Comp Neurol 1999;405:508528.

49. Pencea V, Bingaman KD, Wiegand SJ, Luskin MB. Infusion of brain-derived neurotrophic factor into the lateral ventricle of the adult rat leads to new neurons in the parenchyma of the striatum, septum, thalamus and hypothalamus. J Neurosci 2001;21:6706-6717.

50. Benraiss A, Chmielnicki E, Lerner K, et al. Adenoviral brainderived neurotrophic factor induces both neostriatal and olfactory neuronal recruitment from endogenous progenitor cells in the adult forebrain. J Neurosci 2001;21:67186731

51. Kokaia Z, Zhao Q, Kokaia M, et al. Regulation of brainderived neurotrophic factor gene expression after transient middle cerebral artery occlusion with and without brain damage. Exp Neurol 1995;136:73-88.

52. Arai $S$, Kinouchi $H$, Akabane $A$, et al. Induction of brainderived neurotrophic factor (BDNF) and the receptor trk $\mathrm{B}$ mRNA following middle cerebral artery occlusion in rat. Neurosci Lett 1996;211:57-60.

53. Takami K, Iwani M, Kiyota $\mathrm{Y}$, et al. Increase of basic fibroblast growth factor immunoreactivity and its mRNA level in rat brain following transient forebrain ischemia. Exp Brain Res 1992;90:1-10.

54. Lippoldt A, Andbjer B, Rosén L, et al. Photochemically induced focal cerebral ischemia in rat: time dependent and global increase in expression of basic fibroblast growth factor mRNA. Brain Res 1993;625:45-56.

55. Lin $\mathrm{TN}, \mathrm{Te} \mathrm{J}$, Lee $\mathrm{M}$, et al. Induction of basic fibroblast growth factor (bFGF) expression following focal cerebral ischemia. Mol Brain Res 1997;49:255-265.

56. Lee WH, Clemens JA, Bondy CA. Insulin-like growth factors in the response to cerebral ischemia. Mol Cell Neurosci 1992; 3:36-43

57. Hallett M. Plasticity of the human motor cortex and recovery from stroke. Brain Res Rev 2001;36:169-174.

58. Rijntjes M, Weiller C. Recovery of motor and language abilities after stroke: the contribution of functional imaging. Prog Neurobiol 2002;66:109-122.

59. Arvidsson A, Collin T, Kirik D, Kokaia Z, Lindvall O. Neuronal replacement from endogenous precursors in the adult brain after stroke. Nat Med 2002;8:963-970. 